\title{
Standardization procedure for plasma biomarker analysis in rat models of epileptogenesis: Focus on circulating microRNAs
}

\author{
*'Erwin A. van Vliet, $\nmid$ 'Noora Puhakka, *James D. Mills, $\ddagger$ Prashant K. Srivastava $\$$ Michael R. \\ Johnson, §Paolo Roncon, $\uparrow$ Shalini Das Gupta, $\dagger$ Jenni Karttunen, §đMichele Simonato, \#Katarzyna \\ Lukasiuk, **Jan A. Gorter, *Eleonora Aronica, and †Asla Pitkänen \\ Epilepsia, 58(12):2013-2024, 2017 \\ doi: 10.1111/epi.13915
}

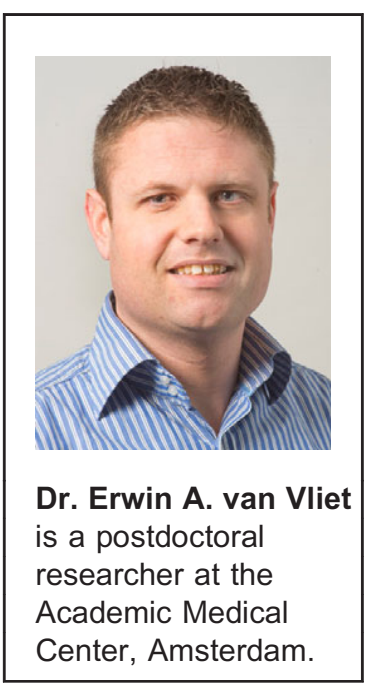

\section{SUMMARY}

The World Health Organization estimates that globally 2.4 million people are diagnosed with epilepsy each year. In nearly $30 \%$ of these cases, epilepsy cannot be properly controlled by antiepileptic drugs. More importantly, treatments to prevent or modify epileptogenesis do not exist. Therefore, novel therapies are urgently needed. In this respect, it is important to identify which patients will develop epilepsy and which individually tailored treatment is needed. However, currently, we have no tools to identify the patients at risk, and diagnosis of epileptogenesis remains as a major unmet medical need, which relates to lack of diagnostic biomarkers for epileptogenesis. As the epileptogenic process in humans is typically slow, the use of animal models is justified to speed up biomarker discovery. We aim to summarize recommendations for molecular biomarker research and propose a standardized procedure for biomarker discovery in rat models of epileptogenesis. The potential of many phylogenetically conserved circulating noncoding small RNAs, including microRNAs (miRNAs), as biomarkers has been explored in various brain diseases, including epilepsy. Recent studies show the feasibility of detecting miRNAs in blood in both experimental models and human epilepsy. However, the analysis of circulating miRNAs in rodent models is challenging, which relates both to the lack of standardized sampling protocols and to analysis of miRNAs. We will discuss the issues critical for preclinical plasma biomarker discovery, such as documentation, blood and brain tissue sampling and collection, plasma separation and storage, RNA extraction, quality control, and RNA detection. We propose a protocol for standardization of procedures for discovery of circulating miRNA biomarkers in rat models of epileptogenesis. Ultimately, we hope that the preclinical standardization will facilitate clinical biomarker discovery for epileptogenesis in man.

KEY WORDS: Brain, Blood, Discovery, Epilepsy, Preclinical.

\footnotetext{
Accepted September 6, 2017; Early View publication September 27, 2017.

*Department of (Neuro)Pathology, Academic Medical Center, University of Amsterdam, Amsterdam, The Netherlands; †A. I. Virtanen Institute for Molecular Sciences, University of Eastern Finland, Kuopio, Finland; †Division of Brain Sciences, Imperial College Faculty of Medicine, London, United Kingdom; §Division of Neuroscience, University Vita-Salute San Raffaele, Milan, Italy; qUniversity of Ferrara, Ferrara, Italy; \#Laboratory of Epileptogenesis, Nencki Institute of Experimental Biology of Polish Academy of Sciences, Warsaw, Poland; and **Swammerdam Institute for Life Sciences, Center for Neuroscience, University of Amsterdam, Amsterdam, The Netherlands

Address correspondence to Asla Pitkänen, A. I. Virtanen Institute for Molecular Sciences, University of Eastern Finland, PO Box 1627, FI-70211 Kuopio, Finland.E-mail: asla.pitkanen@uef.fi

${ }^{1}$ These authors contributed equally.

Wiley Periodicals, Inc.

(C) 2017 International League Against Epilepsy
} 


\section{Key Points}

- We discuss the issues critical for preclinical plasma biomarker discovery

- We propose a protocol for standardization of procedures for discovery of circulating microRNA biomarkers in rat models of epileptogenesis

- We aim to facilitate clinical biomarker discovery for epileptogenesis in man by preclinical standardization

A biomarker is a biological characteristic that is objectively measured and evaluated as an indicator of normal biological or pathological processes, or a response to a therapeutic intervention. ${ }^{1}$ An "ideal" biomarker should be specific, sensitive, reproducible, predictive, and accurate. The detection assay should be noninvasive, robust, standardized, affordable, and easy to perform.

Only a decade ago, microRNAs (miRNAs) were discovered in mammals as a large class of evolutionarily conserved small noncoding RNAs. ${ }^{2}$ miRNAs regulate gene expression at the posttranscriptional level and, by influencing protein translation, they can be powerful regulators of a wide range of biological processes. miRNAs have been recently shown to regulate several key processes involved in the development of epilepsy after brain injury in experimental models and humans. ${ }^{3}$ miRNAs are found in stable forms in the circulating blood, where their levels change in disease states, thereby representing an appealing source of molecules that could serve as biomarkers for diagnosing or predicting the evolution of diseases, for example, the likelihood of an epileptogenic process and the response to therapy. ${ }^{4}$

One of the major challenges in biomarker analysis of central nervous system diseases is a reliable identification of biomarkers that can be analyzed routinely in easily accessible samples such as blood. Recent studies show the feasibility of determining changes of circulating miRNAs in rodent models of diseases, including brain diseases (Table S1). However, comparison of data is challenging, as the methodologies used vary from study to study. For example, protocols for blood sampling, plasma separation, and quantitation of circulating miRNA levels vary. Preanalytical and technical factors can easily affect the miRNA levels, resulting in bias that does not reflect the biological state of the samples. ${ }^{5}$ Therefore, attention to methodological details is critical.

The first step in setting up a pipeline for blood biomarker discovery is to obtain high-quality blood samples (Fig. 1). Standardized sampling protocols have been recommended for human studies, which can be easily adopted in different laboratories in multicenter trials (e.g., https://intbir.nih.gov/ sites/all/themes/nivea//NINDS_Repository_Biomarkers_ Discovery_Samples_Resource_Manual.pdf). The need for powered, harmonized studies has been well recognized also in experimental modeling of human diseases, including biomarker discovery in epilepsy. ${ }^{6-8}$ Here, our objectives were to (1) review the existing literature of plasma sampling protocols in rat and (2) test and compare different plasma sampling protocols, to identify the one that best suits the needs of the search of miRNA biomarkers for epileptogenesis.

\section{DOCUMENTATION}

There are a number of factors that may influence the expression of blood biomarkers (e.g., age, sex, strain, diet, food and water intake, overall health, time of the day of blood withdrawal, stress) that cannot be accounted for without proper prospective documentation. The need for standardization of preclinical studies was recognized in the European Union 7th Framework-funded project Targets and Biomarkers for Antiepileptogenesis (EPITARGET), a consortium of 18 partners in nine European Countries, 12 of which conduct preclinical studies. This led to the design of the first available common data elements (CDEs) for preclinical studies on epilepsy to help investigators to systematically collect, analyze, standardize, and share preclinical epilepsy data. ${ }^{8}$ These CDEs and case report forms can be downloaded from the EPITARGET Web page (www.epitarget.eu) and are highly recommended. The work is being expanded to a wider spectrum of epilepsy models by an International League Against Epilepsy working group. ${ }^{9}$

\section{Blood Sampling Techniques}

Sampling from the lateral tail vein is quick and simple to perform and can be performed repeatedly within the same animal (see https://www.nc3rs.org.uk/rat-tail-vein-non-sur gical). This technique requires the tail to be warmed (e.g., in warm water) to dilate the lateral tail vein prior to taking the sample. Typically, a 23-G needle or butterfly needle is used to collect blood under short isoflurane anesthesia (induction, 5 vol\%; maintenance, 1-2 vol\%).

Other blood sampling techniques have disadvantages, and therefore, are not preferred for blood biomarker research (https://www.nc3rs.org.uk/rat-decisiontree-blood-sampling). For example, blood vessel cannulation requires surgery, and the indwelling tubes may affect blood biomarker levels. Sampling from the jugular vein requires a high degree of competence to avoid moderate or significant harm to the animal. Sampling from the saphenous vein is limited to a small volume (up to $200 \mu \mathrm{L}$ ). Retro-orbital puncture, trunk blood collection, and intracardiac blood sampling should typically be performed as terminal procedures and cannot be performed longitudinally within the same animal. In addition, samples may be mixed with other tissue fluids in trunk blood collection. One needs to consider that biomarkers may be different in samples from different cannulation sites. 
Plasma miRNA Biomarker Discovery Pipeline for Epileptogenesis
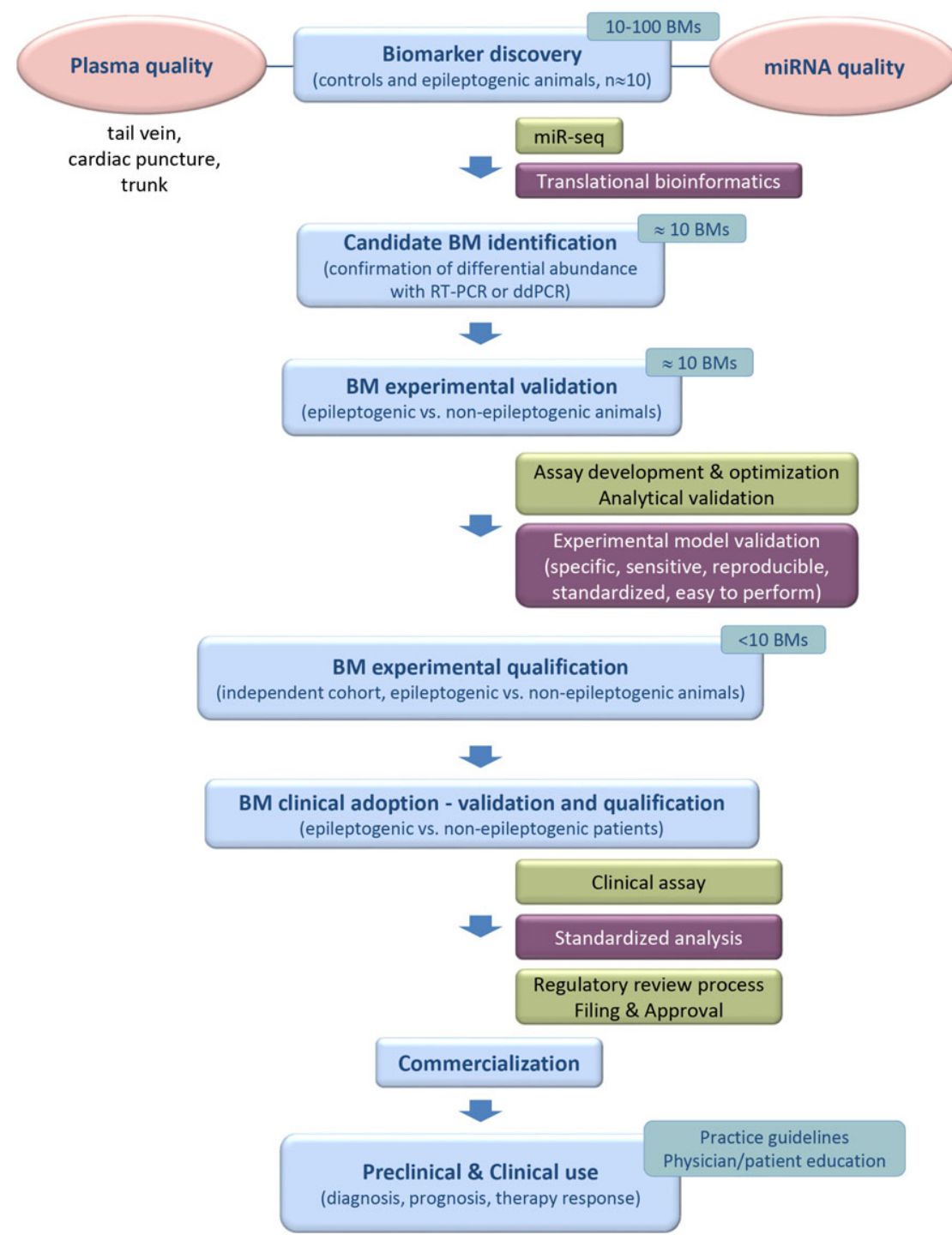

Figure I.

Plasma biomarker discovery pipeline for epileptogenesis. The first step is set up methodologies for high-quality plasma sampling and miRNA analysis as discussed in the text. Phase $I$ - biomarker discovery. In the discovery phase, miRNA sequencing from animals with structural epileptogenic lesions provides tens (or hundreds) of regulated miRNAs as compared to the control group. Bioinformatic analysis reveals their target molecular networks as well as their presence in human miRNome. Phase 2 - candidate biomarker identification. In vitro validation of candidate miRNAs in rat plasma can be done, for example, using real-time polymerase chain reaction (RT-PCR) or droplet digital PCR (ddPCR). One can also assess whether the top candidates are expressed in the brain of the same animal at a given time point. Based on these analyses, the top candidates (about 10) will selected for further analysis. Phase 3-biomarker experimental validation. Levels of top candidate miRNAs will be measured during epileptogenesis in plasma of animals, which are epilepsy-phenotyped with video-electroencephalographic monitoring. Analysis can include several time points and several experimental models of epileptogenesis. Phase 3 can include assay development and optimization. Data analysis includes the definition of biomarker sensitivity and specificity, and determination of miRNA levels, which report on high risk of epileptogenesis. Phase 4-biomarker experimental qualification. Performance of candidate biomarker(s) to diagnose epileptogenesis should be demonstrated in an independent animal cohort using a statistically powered study design. Phase 5-Clinical adoption. Preferably, the etiology of epilepsy in the study population should be comparable to that used in experimental biomarker discovery. Human plasma should be collected in a standardized way, assay methodology should be adapted to human samples, and "normal versus abnormal" levels of a given miRNA in human plasma should be determined. If validation is successful, biomarkers should be taken to the regulatory approval process and commercialization. Phase 6. Eventually, miRNA biomarkers can become part of clinical diagnostic assessment guidelines. miRNA biomarkers can also be used in preclinical and clinical therapy discovery studies. BM, biomarkers; miR-seq, miRNA sequencing.

Epilepsia (C) ILAE 


\section{Blood Volume}

Because the blood volume that can be withdrawn from mice is quite small $(50-200 \mu \mathrm{L})$, rats are more commonly used for biomarker research (Table S1). A search in PubMed using the terms "microRNA," "biomarker," "blood," and "mouse" revealed only four studies in which miRNA biomarkers were studied for diseases of the central nervous system using mice. For rats, the maximum volume of blood that can be obtained on any single occasion is $<10 \%$ of the total blood volume $(\sim 2.56 \mathrm{~mL}$ in a $400-\mathrm{g}$ rat $)$ and $<15 \%$ of the total blood volume $(\sim 3.84 \mathrm{~mL})$ in 28 days. See https://www.nc3rs.org.uk/rat-decision-tree-blood-sa mpling for more details.

\section{Collection Tubes}

Depending on the type of biomarkers and methods used, plasma and/or serum can be obtained. Plasma is the cell-free supernatant that is obtained after centrifugation of blood that has been collected in the presence of an anticoagulant. Serum is the cell-free supernatant obtained after centrifugation of blood that has been collected in the absence of an anticoagulant and allowed to spontaneously clot. The essential difference between plasma and serum is the presence or absence of fibrinogen and clotting factors, respectively. For small noncoding RNAs (including miRNAs), plasma is preferred over serum to avoid (1) procedural variation in clotting and coagulation process and (2) release of small RNAs from blood cells (platelets, white and red blood cells) into serum during the coagulation process. ${ }^{10,11}$

The type of anticoagulant used in plasma collection tubes is also important to consider. Whereas $\mathrm{K}_{2}$ ethylenediaminetetraacetic acid (EDTA) is an acceptable anticoagulant for downstream analysis using quantitative polymerase chain reaction (PCR), the use of heparin or trisodium citrate as an anticoagulant potently inhibits subsequent PCR. ${ }^{11,12}$ The use of tubes containing EDTA in dried format is preferred over those containing EDTA in solution, because if tubes containing a standard volume of EDTA are filled incompletely, the final biomarker concentration is diluted unequally as compared to other samples. Blood and EDTA are mixed by inverting the tube $10 \times 180^{\circ}$ and back.

\section{Centrifugation}

After withdrawal of blood, plasma is separated using centrifugation. Plasma has to be removed with caution to avoid disruption of the buffy coat. Because plasma processing conditions substantially influence circulating biomarker levels, ${ }^{13}$ we recommend cooling the collected blood immediately at $4^{\circ} \mathrm{C}$ and centrifuging it within $1 \mathrm{~h}$ to remove the bulk of platelets at $1,300 \times \boldsymbol{g}$ for $10 \mathrm{~min}$ at $4^{\circ} \mathrm{C}$. At this speed, most platelets will be removed, because much lower centrifugation speeds (e.g., $600 \times g$ ) are used to obtain platelets. ${ }^{13}$ This is important, as platelets have a large repertoire of miRNAs and are the major source of miRNAs in plasma, ${ }^{14}$ which can influence miRNA assessment. However, plasma may still contain residual platelets, ${ }^{13,14}$ and therefore, we recommend a second centrifugation $\left(3000 \times \mathbf{g}, 10 \mathrm{~min}\right.$ at $\left.4^{\circ} \mathrm{C}\right)$ to remove residual platelets. ${ }^{14,15}$ In case archived samples were frozen after the first centrifugation step, the second step can be applied after thawing, which effectively removes platelet contamination from these samples. ${ }^{13}$ However, it should be noted that the residual frozen platelets may have released miRNAs into plasma. $^{14}$

Although miRNAs in extracted plasma appear to be stable at room temperature (at least up to $24 \mathrm{~h}$ ), it is not yet known whether the time interval between blood collection and processing of plasma affects miRNA levels. ${ }^{12}$ Obviously, the optimal approach for miRNA purification from the whole blood is to collect fresh blood and process the sample as quickly as possible, and not later than 2-4 h after collection. $^{11}$

\section{Storage}

It has been reported that multiple freeze-thaw cycles have no effect on reproducibility of miRNA profiling from plasma. ${ }^{11,12}$ Nonetheless, it seems wise to avoid repeated freeze-thaw cycles by freezing collected plasma immediately in smaller aliquots using dry ice and storing the samples at -70 to $-80^{\circ} \mathrm{C}$. ${ }^{12}$ Stability of blood miRNAs is due to several protective mechanisms, including association with plasma proteins, specifically argonaute-2 or lipoproteins, or encapsulation in extracellular vesicles. ${ }^{11}$ Extracted miRNAs are very stable when stored at $-70^{\circ} \mathrm{C}$ for 1 year. ${ }^{16}$ Although specimens have been used that are several years old, the effect of long-term storage on miRNA levels has not yet been comprehensively evaluated. Thus, it is best to match case and control specimens with respect to duration of storage as much as possible. ${ }^{12}$

\section{Plasma Quality Control}

Poor plasma quality is typically caused by technical reasons related to difficulties in blood withdrawal (e.g., dehydration of animal, poor sampling technique/equipment) or consequent plasma preparation in a given epilepsy model. However, it is a striking observation that only 4\% (two of 43) of published rat studies (Table S1) assessing miRNA in rat plasma have used any quality measurements to assess their sampling protocol performance.

There are several protocols that can be used for quality control of rat plasma (Table 1). As discussed in the previous paragraph, residual platelets that are present in plasma may influence miRNA levels. Therefore, a proper quality control for the presence of platelets should be performed. Similarly, hemolyzed erythrocytes may also release miRNAs, which 


\begin{tabular}{|c|c|c|c|c|c|}
\hline Purpose & Description & Sample amount, $\mu \mathrm{L}$ & Instrument & Cutoff value & References \\
\hline Hemolysis & Visual inspection & - & - & Red color & 18,19 \\
\hline Hemolysis & $\begin{array}{l}\text { Absorbance of } \\
\text { hemoglobin at } 414 \mathrm{~nm}\end{array}$ & 1.0 & Nanodrop- 1000 or similar & $0.20-0.25$ & 19,20 \\
\hline Hemolysis & $\begin{array}{l}\text { Ratio of red blood cell- } \\
\text { enriched miR- } 45 \text { I to the } \\
\text { reference miR-23a }\end{array}$ & $\begin{array}{l}50-200 \\
\text { (for RNA extraction) }\end{array}$ & $\begin{array}{l}\text { Real-time PCR system } \\
\text { (Applied Biosystems) or similar }\end{array}$ & $\Delta \mathrm{C}_{\mathrm{q}}=5$ & 5,18 \\
\hline $\begin{array}{l}\text { Platelet } \\
\text { contamination }\end{array}$ & $\begin{array}{l}\text { Ratio of platelet-enriched } \\
\text { miR-425 to the reference miR-23a }\end{array}$ & $\begin{array}{l}50-200 \\
\text { (for RNA extraction) }\end{array}$ & $\begin{array}{l}\text { Real-time PCR system } \\
\text { (Applied Biosystems) or similar }\end{array}$ & $\Delta \mathrm{C}_{\mathrm{q}}=-\mathrm{I}$ & 5 \\
\hline
\end{tabular}

\begin{tabular}{|ll|}
\hline \multicolumn{1}{|c|}{ Table 2. Protocol for circulating biomarkers from rat } \\
plasma
\end{tabular}

may lead to false discovery of disease-associated biomarkers. ${ }^{5,17-19}$ For human samples, Shah et al. ${ }^{18}$ have compared several methods for detecting hemolysis in serum samples, such as visual inspection, measurement of hemoglobin absorbance by spectrophotometry at $414 \mathrm{~nm}$, measurement of hemoglobin using a Coulter AcT diff Analyzer, and the ratio of red blood cell-enriched miR-451 to the reference miR-23a. They reported that the miRNA ratio was the most sensitive indicator of hemolysis, and therefore, was recommended.

An alternative method is to measure hemoglobin at $414 \mathrm{~nm}$ and use a cutoff absorbance value of 0.20 $0.25{ }^{18-20}$ Although not a typical problem in rat samples, it should be noted that lipemia in plasma interferes with hemoglobin absorbance, which may cause an increase in absorbance values, even in the absence of any hemoglobinrelated peak. ${ }^{21}$ By also measuring the plasma absorbance at $385 \mathrm{~nm}$ (as a lipemia indicator) it is possible to identify hemolyzed samples, independent of lipemia. ${ }^{21}$

Blondal et al. ${ }^{5}$ suggested that a $\Delta \mathrm{Cq}$ value of $>5$ for the miRNA ratio indicator (miR-23a - miR-451) implies possible erythrocyte miRNA contamination, and a $\Delta \mathrm{Cq}$ of $7-8$ or more indicates a high risk of hemolysis in human samples. According to our analysis, the reference values suggested for human serum/plasma hemolysis appear to remain relevant for rat plasma (Fig. 2A1). In addition, we were able to extend previous findings by showing that hemolysis in the sample could also indicate platelet contamination. The ratio of platelet-enriched miR-425 to reference miRNA miR-23a correlated with erythrocyte contamination due to hemolysis $(r=0.599, p<0.01$, Fig. 2A2,B). Absorbance of hemoglobin in some samples indicated values $>0.25$ (Fig. 2C), which was set as an upper limit. ${ }^{18-20}$

Our results suggest that absorbance of hemoglobin is a more sensitive method for rat plasma than the ratio of red blood cell-enriched miR-451 to miR-23a. In addition, after brief practical training, hemoglobin absorbance correlated well with the visual inspection of the plasma color. Interestingly, when samples were freeze-thawed and centrifuged $(3,000 \times \boldsymbol{g})$, the miR-425:miR-23a ratio highly correlated with erythrocyte contamination due to hemolysis $(r=0.599, p<0.01$, Fig. 2D,E). These data suggest that a simple visual inspection accompanied with absorbance measurement of hemoglobin could indicate the overall rat plasma quality for miRNA studies when an upper limit for absorbance is set to 0.25 . When this upper limit was used for plasma samples obtained at different time points after electrically induced status epilepticus (1 day, 1 week, 34 months; $\mathrm{n}=5-6$ for each time point), none of the samples had to be excluded (unpublished observations).

Platelet contamination can confound the measurement of extracellular miRNAs, especially in archived plasma, if only one centrifugation at a relatively low speed is performed. Further studies with more thorough analysis of hemolysis and platelet markers, for example, using a 
combination of multiple methods (or miRNAs) with a larger number of animals, is needed to replicate and validate these findings.

\section{RNA EXTRACTION}

An underappreciated variable that can affect miRNA quantification is the volume of plasma used for small RNA extraction. ${ }^{22}$ Therefore, it is recommended to optimize plasma volume for accurate quantification; too little plasma will have insufficient miRNA for quantification, and too much plasma can increase the concentrations of endogenous polymerase inhibitors (e.g., hemoglobin, lactoferrin, or $\operatorname{IgG}){ }^{22} \mathrm{In}$ one study, it was observed that $50 \mu \mathrm{L}$ of human serum or plasma improved detection of miRNA by PCR as compared to 10 or $200 \mu \mathrm{L} .{ }^{23}$ Another study reported that $200 \mu \mathrm{L}$ of human plasma was to be preferred over 250, 300, or $500 \mu \mathrm{L} .{ }^{16} \mathrm{We}$ therefore recommend using $50-200 \mu \mathrm{L}$ of rat plasma for RNA extraction.

Several other factors during RNA extraction may also affect miRNA quantification. A recent study using cell cultures and human plasma showed that miRNA recovery was largely influenced by the isolation method and by the amount of input material used. ${ }^{24}$ RNA extraction methods can be roughly classified into three main groups: (1) phenol-based techniques that rely on the use of organic solvents, phase separation, and recovery of RNA by precipitation; (2) combined phenol and column-based techniques that utilize phenol and chloroform to separate RNA from other constituents, and a column for RNA adsorption; and (3) column-based techniques that use a phenol-free lysis buffer and a column for RNA recovery. ${ }^{24}$ These three techniques were evaluated by comparing phenol-based (TRIzol LS; Thermo Fisher Scientific), two column-based (miRCURY RNA Isolation and miRCURY biofluids kits; Exiqon), and combined phenol- and column-based (miRNeasy Mini kit; Qiagen) RNA isolation techniques. ${ }^{24}$ The miRCURY kit permitted isolation of highly pure RNA, of better quality when compared to miRNeasy and TRIzol LS; however, miRCURY columns tended to be saturated by large RNA species when the RNA input was increased, which dramatically affected the recovery of miRNAs. ${ }^{24}$ Interestingly, despite the low purity of TRIzol LS-extracted RNA, this method outperformed the miRCURY kit when using high-input material. ${ }^{24}$ Importantly, when using low-input material, the miRNeasy kit permitted better miRNA detection when compared to the miRCURY kit. ${ }^{24}$ The ability to recover miRNAs from 100 and $200 \mu \mathrm{L}$ of human plasma was comparable between miRNeasy and miRCURY kits, whereas the detection of miRNAs from $50 \mu \mathrm{L}$ was better achieved with miRNeasy. Sourvinou et al. ${ }^{16}$ showed that the mirVana PARIS kit and miRNeasy Mini Kit produced the highest yield of recovery for a spike-in miRNA compared to TRIzol extraction in human plasma samples, with the first kit also demonstrating better performance than miRNeasy. However, these results were in contrast to those obtained by Kroh et al. ${ }^{12}$ demonstrating that RNA extraction with miRNeasy led to a two- to threefold increase in RNA yield compared to mirVana in human samples. In another study, TRIzol LS, mirVana PARIS, and miRNeasy kits were compared using human samples. ${ }^{15}$ The mirVana kit and the miRNeasy kit performed better than TRIzol extraction, due to the presence of organic and phenolic contaminants in the TRIzolextracted RNA. ${ }^{15}$ The miRNeasy kit had more stable RNA yields through different extraction days. ${ }^{15}$ Similarly, Page et al. ${ }^{25}$ compared three different commercial kits for RNA extraction using human samples and demonstrated that the miRNeasy kit produced the best quantity and quality of circulating miRNAs.

Adding an RNA carrier before RNA extraction may improve quantity and purity of extracted RNA. ${ }^{15,24}$ However, added RNA carrier can mask the quantity of extracted RNA and affect accuracy in quantification and quality analysis. ${ }^{15}$

\section{RNA Quality Control}

There is no straightforward method to determine the quality of miRNAs extracted from plasma. A high RNA integrity number (RIN) would suggest contamination with mRNA (and miRNA) derived from circulating cells. Typically, the concentration and quality of RNA obtained after extraction is measured using a NanoDrop spectrophotometer. Although this can be used to assess the presence of contaminants such as peptides, phenols, aromatic compounds, or carbohydrates and proteins, these methods do not provide sufficient information about the concentration and quality after extraction of small noncoding RNAs from plasma, due to the low concentration of miRNAs. ${ }^{15,21,24}$ Alternatively, a Bioanalyzer (Agilent Technologies, Palo Alto, CA, U.S.A.), which utilizes a highly sensitive, chip-based assay, could be used to detect small amounts of RNA. However, it is very difficult to differentiate miRNAs from all other types of small RNAs or from possible fragments produced by degraded mRNA transcripts. This may be improved by using a Fragment Analyzer (Advanced Analytical Technologies, U.S.A.). However, not every laboratory is equipped with this apparatus. Generally, a fixed volume of input material rather than an equal quantity of RNA is recommended after extraction of small noncoding RNAs for plasma. ${ }^{21}$

\section{DETECTION OF MIRNAS AND NORMALIZATION}

For rat plasma, so far, real-time PCR has been the most common method to assess miRNA levels $(80 \%$ of studies, 36 of 43, Table S1). Other platforms include the sequencing 


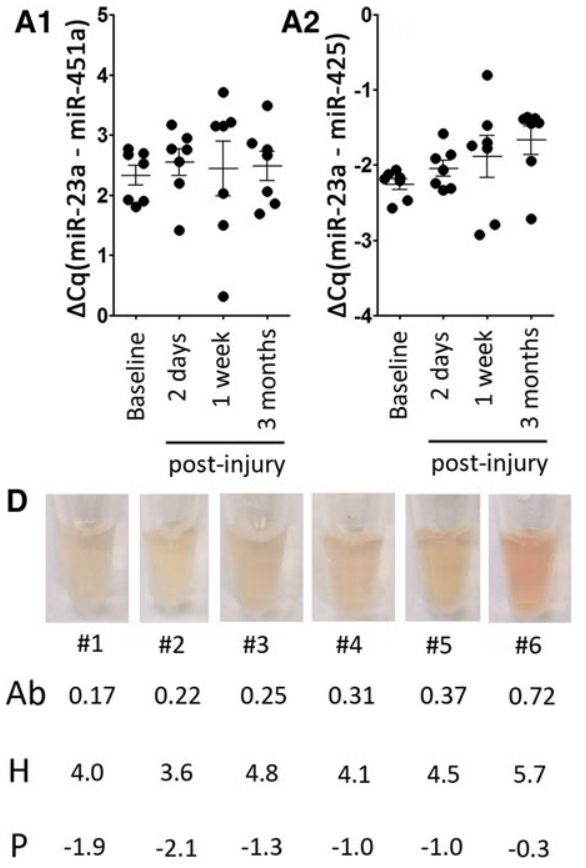

B

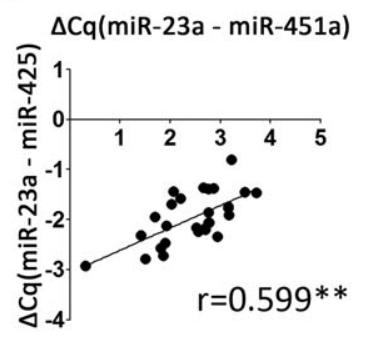

C

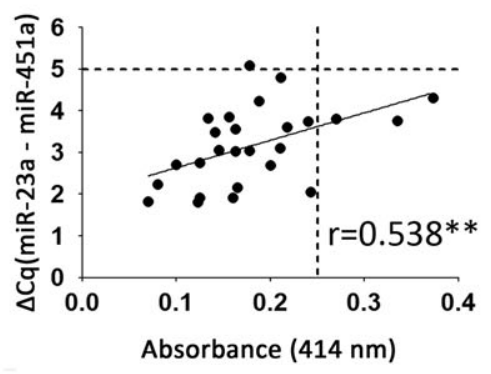

E3

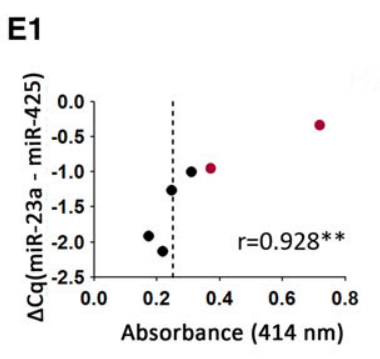

E2

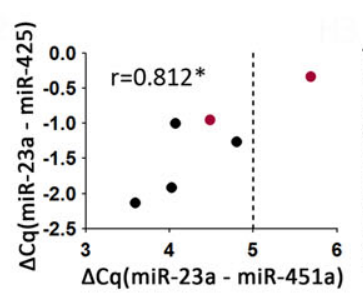

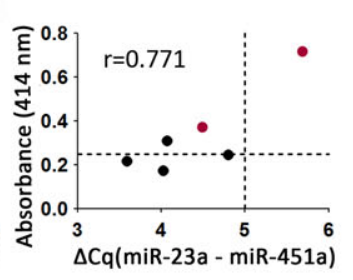

Figure 2.

Hemolysis and platelet contamination in rat plasma obtained by tail vein withdrawal and standardized plasma separation procedure. (AI) Hemolysis contamination. Ratio of red blood cell-enriched miR-45 la to the reference miRNA miR-23a in samples obtained at 7 days (baseline) before traumatic brain injury (TBI) and at 2 days, 7 days, or 3 months post-TBI. The miR-45 la:miR-23a ratio did not differ between the time points, indicating reliable sampling $(p>0.05$, Mann-Whitney $U$ test). Each time point included three control and four $\mathrm{TBI}$ rats (no difference between the groups, $\mathrm{p}>0.05$, Mann-Whitney $U$ test). Importantly, all samples had a $\Delta \mathrm{Cq}$ value $<5$. No visual hemolysis (red color) was detected among the samples. (A2) Platelet contamination. A ratio of platelet-enriched miR-425 to the reference miRNA miR-23a in the same samples as presented in Al is shown. The miR-425:miR-23a ratio did not differ between the time points or groups, indicating reliable sampling $(p>0.05$, Mann-Whitney $U$ test). Our data suggest that samples having $\Delta C q(m i R-23 a-m i R-425)$ close to or above 0 have a high risk of platelet contamination. (B) Correlation between sample contamination by red blood cells and platelets $(r=0.599, p<0.01$, Spearman rank). (C) Correlation between $\Delta C q(m i R-23 a-$ miR-45la) and absorbance of hemoglobin $(r=0.538, p<0.0$ I, Spearman rank). Plasma $(n=26)$ was sampled at 7 days (baseline) prior TBI, and at 2 or I4 days post-TBI. No difference was observed in $\Delta \mathrm{Cq}(\mathrm{miR}-23 \mathrm{a}-\mathrm{miR}-45 \mathrm{la})$ or absorbance of hemoglobin between the time points, indicating reliable sampling $(p>0.05$, Mann-Whitney $U$ test). Each time point included both control and TBI rats (no difference between groups [p $>0.05]$, MannWhitney $U$ test). (D) Series of plasma samples investigated with visual inspection, using a spectrophotometer (4I4 nm), and real-time quantitative polymerase chain reaction (RT-qPCR; miR-23a, miR-45 Ia, and miR-425). First, samples $(n=6)$ were ordered by an experienced researcher according the color from yellowish to the most red-colored sample. Then, hemoglobin absorbance was measured using a spectrophotometer. With visual inspection, the researcher was able to indicate that samples \#5 and \#6 were hemolyzed. Spectrophotometric A4I4-nm analysis suggested that the fourth sample also had a significant hemoglobin content ( $>0.25)$. On the other hand, RT-qPCR indicated that only the sixth sample was hemolyzed, according the guidelines for human samples $(\Delta \mathrm{Cq}[\mathrm{miR}-23 \mathrm{a}-\mathrm{miR}-45 \mathrm{Ia}]>5)$. In light of these data, we suggest that visual inspection accompanied with spectrophotometric (A4I4-nm) measurement of hemoglobin is the best approach to assess hemolysis in plasma samples from rat tail vein blood. Importantly, the hemolysis measurement indicated the overall sample quality, as we found a relationship between the absorbance of hemoglobin with miR-45 Ia (indicator of red blood cells) and with miR-425 (indicator of platelets). (EI-E3). Correlations between the measured parameters in panel D. Hemoglobin absorbance was positively correlated with miR-425 content (platelet marker) in the sample (EI; $r=0.928, p<0.0$ I, Spearman rank). Furthermore, the level of erythrocyte marker miR-45 Ia was positively correlated with mir-425 content (E2; $r=0.8 I 2, p<0.05$, Spearman rank). In addition, we observed a trend for a correlation between the ratio of red blood cell-enriched miR-45I to the reference miR-23a and hemoglobin absorbance (E3; $r=0.77 \mathrm{I}, \mathrm{p}=0.072$, Spearman rank). Dotted lines represent the cutoff values suggested for human samples (see text for references). Red data points indicate the samples with hemolysis in visual inspection (fifth and sixth) in panel $D$. Ab, absorbance of hemoglobin; $\mathrm{H}$, contamination by red blood cells; $\mathrm{P}$, contamination by platelets; $r$, Spearman correlation coefficient; $\Delta \mathrm{Cq}$, delta quantification cycle; ${ }^{*} \mathrm{p}<0.05 ; * * \mathrm{p}<0.01$

Epilepsia (C) ILAE

(4\%, two of 43), microarray (7\%, three of 45), and Firefly ( $2 \%$, one of 43 ) techniques. Three studies evaluated here did not report the platform they used (7\%, three of 43$)$.
For optimal quantification of plasma miRNAs, it is important to consider the whole workflow starting from sample processing. ${ }^{26}$ Later decision steps highlight the 
importance of reduction of technical variation and choice of PCR primers ${ }^{27}$ to be able to minimize the need of downstream normalization procedures. For rats, the most popular reverse transcriptase and PCR system is usage of stem-loop miRNA-specific reverse transcription oligonucleotides and PCR probes (56\% of all PCR studies). For quantitative PCR normalization, plasma biomarker studies have used multiple strategies (Table S1), and normalization after PCR is still heavily used in comparison to efforts to minimize technical variation prior to PCR. Overall, the most common method is relative quantification normalized to external spike-in (58\%, 21 of 36; e.g., cel-miR-39, cel-miR-2, ath-miR159a). Other methods are normalization to U6 or one or multiple stable endogenous miRNA(s), normalization using global mean normalization, or absolute quantification with synthetic miRNA standard curve (which could be recommended for real-time PCR if efforts to minimize technical variability are performed). It is recommended to use a set of spike-in templates during RNA isolation combined with a carefully selected set of assays targeting endogenous miRNAs. ${ }^{5}$ Because PCR efficiency and normalization method can have a massive impact on final real-time PCR results, efforts to minimize technical variation and creation of intraand interassay controls as well as usage of digital PCR for absolute quantification could have promise when developing miRNA biomarker platforms using rat plasma.

\section{Next Generation Sequencing}

Over the past decade, the development of various next generation sequencing techniques has revolutionized research in molecular genomics and transcriptomics. One such sequencing technique known as RNA sequencing (RNA-Seq), has surpassed microarrays as the gold-standard for gene-expression studies. ${ }^{28}$ Unlike microarrays, which use probe hybridization technology to quantify gene expression over a series of predefined probes, RNA-Seq produces a digital signal from a cDNA library input. Apart from quantifying gene expression levels and detecting differentially expressed genes, RNA-Seq can also be used to explore alternative splicing events, detect novel transcripts, discover gene fusion events, map transcription start sites, and identify sequence variation in transcribed regions. ${ }^{28-30}$ RNASeq also has a lower level of background noise, can detect low-abundance transcripts, and requires a lower concentration of input RNA than microarrays. ${ }^{28,29}$ RNA-Seq has been utilized to successfully detect miRNAs and other small RNAs in rat (unpublished data), bovine, and human plasma. ${ }^{31-34}$

In most cases, library preparation of the extracted RNA and the subsequent sequencing are completed at a commercial sequencing facility in accordance to their in-house protocols. To reduce the possibility of the introduction of any technical variation during these steps, experimental design should be discussed with the sequencing facility prior to the initiation of the project. Previously, when performing small RNA-Seq on RNA extracted from serum, we have utilized the Illumina TruSeq small RNA-Seq sample preparation kit (Illumina, San Diego, CA, U.S.A.). This method of library preparation will capture miRNAs, other small RNA species, and novel small RNAs. Although sequencing itself is a rather trivial process, it is known that lane and flow cell biases exist during sequencing. ${ }^{35}$ Thus, it is important to make sure that samples are pooled and/or split across lanes, and when possible, all sequenced on the same flow cell. Furthermore, sequencing facilities will generally not randomize sample order and will simply process the samples in the order received. Hence, randomization of sample order prior to collection by the sequencing facility is paramount. It is important to note here that any biases introduced during the RNA isolation or library preparation stage will be enriched during the sequencing process. ${ }^{36}$ For gene expression studies of moderately to highly expressed miRNAs as little as 1-2 million reads, single reads per sample may be sufficient; however, for the detection of lowly expressed miRNAs, novel short RNAs, other RNA species, and isomiRs, a much greater read depth is required (e.g., 12 million reads).

\section{Bioinformatics Analysis of Rat Plasma mirna Data}

Although RNA-Seq is widely utilized throughout the scientific research community, no optimal data analysis pipeline exists. This is further confounded by the existence of a large body of literature outlining different workflows or analysis techniques each purporting to offer a superior, more "true" representation of the transcriptome. In reality, there is no one optimal method for the generation and analysis of RNA-Seq data, and the techniques utilized are often dependent on the biological questions being asked and the personal preference of the bioinformatician analyzing the data (for reviews and comparisons please see Conesa et al., ${ }^{37}$ Buschmann et al., ${ }^{38}$ and Soneson and Delorenzi ${ }^{39}$ ).

The standard RNA-Seq data analysis workflow consists of quality control, mapping of reads, quantification of genes or transcripts, and differential expression testing, followed by pathway or gene ontology enrichment analysis. As part of good experimental design, the type of analysis should be decided before the samples are sent for sequencing. A basic work flow to identify differentially expressed genes is as follows: (1) mapping of reads to the organism reference genome using STAR, Bowtie2, or Burrow-Wheeler Aligner; (2) quantification of reads that map to the location of each miRNA or other short RNA species through FeatureCounts or htseq-count; and (3) differential expression testing using EdgeR or DESeq2 to identify genes of interest. More advanced analysis methods such as various machinelearning techniques ${ }^{40,41}$ can also be taken advantage of to identify genes or transcripts of interest for further investigation. It is essential that selected miRNAs of interest are 
validated using a secondary technique such as real-time or digital PCR.

\section{Comparing MiRNAS IN BRAIN Tissue and Plasma during EPILEPTOGENESIS}

The great advantage of using animal models of epileptogenesis is the availability of brain tissue for analysis. To determine how changes in circulating miRNAs compare with the changes in the brain, one has to determine their expression in plasma as well as in the brain. Moreover, one needs to assess the possible contribution of peripheral organs to plasma miRNA levels during epileptogenesis. In a recent study, we showed that the expression of miR-21 in plasma reflects changes in the hippocampus and parahippocampal cortex during epileptogenesis in the electrical post-status epilepticus rat model. ${ }^{42}$ However, the pattern of expression of miR-146a and miR-142 in plasma was different from brain. miR-146a showed a tendency toward increased expression during the chronic phase in plasma, whereas in hippocampal subregions it was increased during the latent phase. miR-142 showed increased expression in plasma during the acute phase, but its expression was increased in brain during the latent phase. ${ }^{42}$ In another recent study, the expression of miRNAs in plasma and hippocampal granule cells was investigated during epileptogenesis after pilocarpine-induced status epilepticus in rats. ${ }^{43}$ Although 27 miRNAs were differentially expressed in plasma, the only miRNA dysregulated both in plasma and brain was miR-142. However, miR-142 was down-regulated during the chronic phase and up-regulated during the latent phase in granule cells. ${ }^{43}$ In contrast, Hu et al ${ }^{44}$ showed that the expression profile of miR-21, miR-22, miR-34a, and miR-125a was similar in blood and hippocampus after pilocarpine-induced status epilepticus. Also during the acute phase after kainic acid-induced status epilepticus, the expression patterns of several miRNAs were similar between blood and brain (e.g., up-regulation of miR-298 and down-regulation of miR-155, miR-29c, miR-34b, miR-98, miR-122, miR-155, miR-203, and miR-450a). ${ }^{45}$

Because the method used for processing brain tissue may influence expression of (small) RNAs, we assessed RNA and miRNA quality after sampling rat brain tissue using two preservation methods: AllProtect tissue reagent (Qiagen) and snap-freezing in liquid nitrogen (Fig. 3). We also assessed the effect of time delay between decapitation and sample preservation $(5,10$, and $30 \mathrm{~min}$ before preservation, $\mathrm{n}=7$ for each time point) on sample quality. Briefly, macromolecules were isolated from the cerebral cortex using AllPrep DNA/RNA/Protein Mini kit (Qiagen, Cat No./ID: 80004) according to the manufacturer's instructions with few exceptions. To remove excess lipids, $20 \mathrm{mg}$ PHML LIPOSORB Absorbent (Millipore) was added before lysis with TissueLyzer. The reagent was removed by centrifugation. Small RNA was collected from the protein fraction with additional protocol using RNeasy MinElute cleanup kit (Qiagen, Cat No./ID: 74204). The extraction was made in five batches.

\section{Total RNA quality}

The RIN is a commonly used way to assess the integrity of RNA in brain based on the ratio of $28 \mathrm{~S}$ and $18 \mathrm{~S}$ ribosomal RNA. ${ }^{46}$ An acceptable RIN cutoff for downstream assays is controversial, but in general RIN $>8$ is considered to indicate high-quality RNA. ${ }^{47,48}$ Furthermore, Illumina recommends using $\mathrm{RIN} \geq 8$ for sequencing samples.

Analysis of total RNA quality (RIN, Agilent Bioanalyzer RNA 6000 Nano Kit) indicated that samples preserved with snap-freezing had a $0.54 \mathrm{U}$ higher mean and $0.6 \mathrm{U}$ higher median RIN number as compared to samples preserved with AllProtect reagent (Fig. 3A). The median RIN value was $0.35 \mathrm{U}$ lower in the 30-min group than in the 5- or 10-min groups, although the difference was not significant (Fig. 3B). Although we did not detect any major effect of delay between decapitation and tissue preservation on RIN, all snap-frozen RNA samples from 5- to 10-min time points had RIN $>8$. However, in the 30-min batch a few samples had RIN $<8$. When AllProtect reagent was used, several samples at all time points had RIN $<8$, indicating lowerquality RNA.

\section{miRNA yield}

Using lab-on-chip technology, it is possible to quantify miRNAs as a percentage of small RNAs. The miRNA/small RNA ratio is negatively correlated with RIN values, and can be used to estimate the miRNA yield. ${ }^{49}$ Analysis of the miRNA yield (using the Agilent Bioanalyzer 2100 Small RNA kit) indicated that snap-frozen samples had a lower (i.e., better) mean miRNA/small RNA ratio (Fig. 3D). The miRNA/small RNA ratio increased when the preservation was delayed from 5 to $10 \mathrm{~min}$. However, there was no consistent progression in the increase in miRNA/small RNA ratio when the delay was prolonged to $30 \mathrm{~min}$ (Fig. 3E). Analysis of day-to-day variability between the purification batches indicated a higher miRNA/small RNA ratio in day 5 extraction as compared to others (Fig. 3F). The 5-day purification batch included only 10-min samples, which might partly explain the higher miRNA/small RNA ratio.

Like the total RNA quality justified by using the RIN, the miRNA yield justified by using the miRNA/small RNA ratio was also superior in snap-frozen samples as compared to those preserved using AllProtect reagent. That purification of miRNA is a delicate procedure was revealed by a difference in miRNA quality between extraction days (Fig. 3F). It is also possible that use of separate extraction procedures for purification of RNA and small RNAs contributed to variability in miRNA/small RNA ratio. 
A
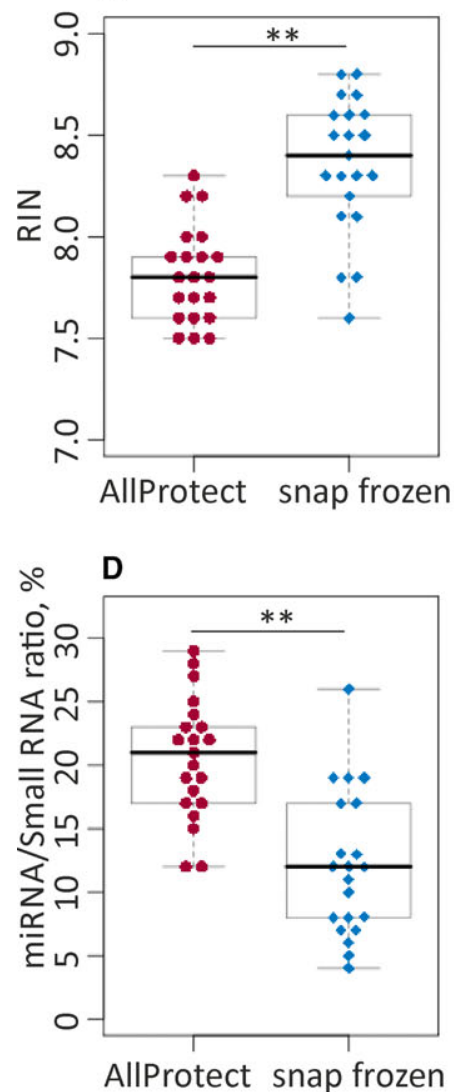

B

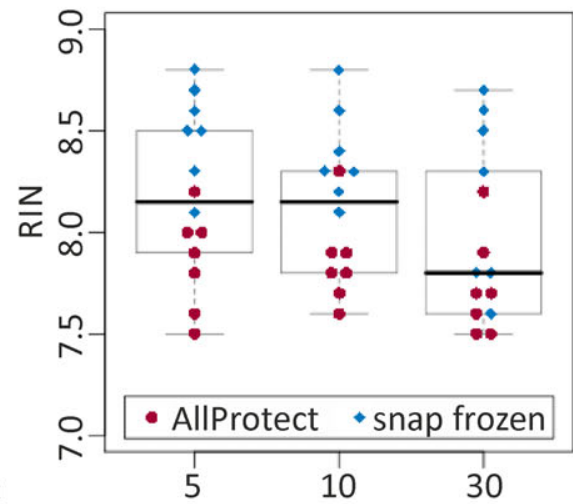
time before preservation, $\min$ E

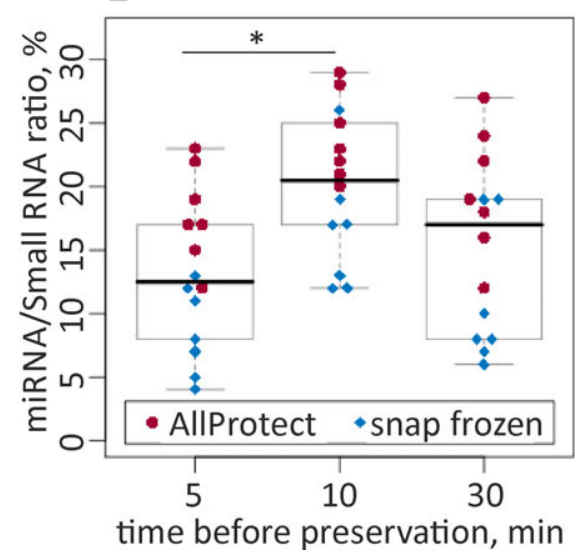

C

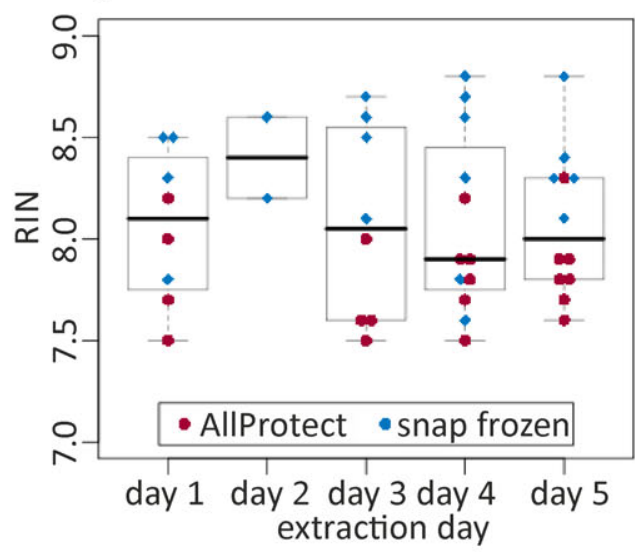

$\mathbf{F}$

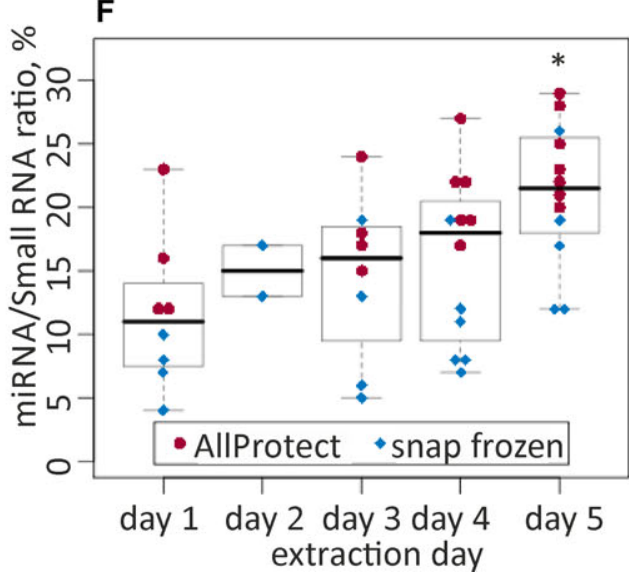

Figure 3.

Comparison of RNA quality obtained from brain samples using different preservation methods. Box plots of RNA integrity number (RIN) for different (A) preservation methods, (B) time points, and (C) extraction days. Snap-freezing with liquid nitrogen is recommended over AllProtect when 2-mm brain slices are sampled. Furthermore, our experiment suggested that the RNA quality is better when samples were obtained within 10 min after decapitation. Boxplots of miRNA/small RNA ratio for (D) preservation method, (E) time points, and (F) extraction days. Similarly, snap-freezing produced better quality of samples. In addition, one extraction day differed from others, indicating possible complication in the purification process. ${ }^{*} \mathrm{p}<0.05$, ${ }^{*} \mathrm{p}<0.0 \mathrm{I}$ calculated using Mann-Whitney $U$ test.

Epilepsia (C) ILAE

We recommend that rat brain tissue samples be preserved with snap-freezing in liquid nitrogen within 10 min after decapitation. The delay between decapitation and tissue preservation should be monitored and reported. Our data show that snap-freezing was a slightly better way to preserve tissue samples, according to the RIN and the miRNA/ small RNA ratio.

\section{Conclusions}

Circulating small noncoding RNAs, particularly miRNAs, hold great promise as minimally invasive biomarkers. Although there is growing attention to biomarker studies, particularly in the preclinical field, there is a lack of consistency and standardization. Therefore, we present a standardization procedure to detect circulating miRNAs in rat plasma (Table 2 and appendix). Once consensus procedures have been defined and applied, studies conducted in different laboratories can be expected to become more comparable, which will facilitate the identification and reproducible use of biomarkers. Ultimately, this will lead to novel diagnostic tests from easily assessable biofluids, such as blood, and provide more insight into the disease mechanisms, which is important for developing novel therapies for the treatment of epileptogenesis in patients at risk.

\section{Acknowledgments}

We thank Anatoly Korotkov, Niina Vuokila, and Anu Lipsanen for their contribution. The research leading to these results has received funding from the European Union's Seventh Framework Program (FP7/2007-2013) under grant agreement No. 602102 (EPITARGET; Erwin A. van Vliet, Michael R. Johnson, Michele Simonato, Katarzyna Lukasiuk, Jan A. Gorter, Eleonora Aronica, Asla Pitkänen), the Dutch Epilepsy Foundation, project number 16-05 (Erwin A. van Vliet), and the European Union's Horizon 2020 Research and Innovation Program under the Marie Sklodowska-Curie grant agreement No. 642881 (Eleonora Aronica, Asla Pitkänen). 


\section{Disclosure}

The authors declare no conflicts of interest. We confirm that we have read the Journal's position on issues involved in ethical publication and affirm that this report is consistent with those guidelines.

\section{REFERENCES}

1. Strimbu K, Tavel JA. What are biomarkers? Curr Opin HIV AIDS 2010;5:463-466.

2. Almeida MI, Reis RM, Calin GA. MicroRNA history: discovery, recent applications, and next frontiers. Mutat Res 2011;717:1-8.

3. Henshall DC, Hamer HM, Pasterkamp RJ, et al. MicroRNAs in epilepsy: pathophysiology and clinical utility. Lancet Neurol 2016; $15: 1368-1376$

4. Pitkanen A, Loscher W, Vezzani A, et al. Advances in the development of biomarkers for epilepsy. Lancet Neurol 2016;15:843-856.

5. Blondal T, Jensby Nielsen S, Baker A, et al. Assessing sample and miRNA profile quality in serum and plasma or other biofluids. Methods 2013;59:S1-S6.

6. Landis SC, Amara SG, Asadullah K, et al. A call for transparent reporting to optimize the predictive value of preclinical research. Nature 2012;490:187-191.

7. Simonato M, Brooks-Kayal AR, Engel J Jr, et al. The challenge and promise of anti-epileptic therapy development in animal models. Lancet Neurol 2014;13:949-960.

8. Lapinlampi N, Melin E, Aronica E, et al. Common data elements and data management: remedy to cure underpowered preclinical studies. Epilepsy Res 2017;129:87-90.

9. Harte-Hargrove LC, French JA, Pitkänen A, et al. Common data elements for preclinical epilepsy research: standards for data collection and reporting. A report of the TASK3 group of the AES/ILAE Translational Task Force of the ILAE. Epilepsia 2017. In press

10. Wang K, Yuan Y, Cho JH, et al. Comparing the MicroRNA spectrum between serum and plasma. PLoS One 2012;7:e41561.

11. Moldovan L, Batte KE, Trgovcich J, et al. Methodological challenges in utilizing miRNAs as circulating biomarkers. J Cell Mol Med 2014;18:371-390.

12. Kroh EM, Parkin RK, Mitchell PS, et al. Analysis of circulating microRNA biomarkers in plasma and serum using quantitative reverse transcription-PCR (qRT-PCR). Methods 2010;50:298-301.

13. Cheng HH, Yi HS, Kim Y, et al. Plasma processing conditions substantially influence circulating microRNA biomarker levels. PLoS One 2013;8:e64795.

14. Mitchell AJ, Gray WD, Hayek SS, et al. Platelets confound the measurement of extracellular miRNA in archived plasma. Sci Rep 2016;6:32651.

15. Moret I, Sanchez-Izquierdo D, Iborra M, et al. Assessing an improved protocol for plasma microRNA extraction. PLoS One 2013;8:e82753.

16. Sourvinou IS, Markou A, Lianidou ES. Quantification of circulating miRNAs in plasma: effect of preanalytical and analytical parameters on their isolation and stability. J Mol Diagn 2013;15:827-834.

17. Pritchard CC, Kroh E, Wood B, et al. Blood cell origin of circulating microRNAs: a cautionary note for cancer biomarker studies. Cancer Prev Res 2012;5:492-497.

18. Shah JS, Soon PS, Marsh DJ. Comparison of methodologies to detect low levels of hemolysis in serum for accurate assessment of serum microRNAs. PLoS One 2016;11:e153200.

19. Kirschner MB, Kao SC, Edelman JJ, et al. Haemolysis during sample preparation alters microRNA content of plasma. PLoS One 2011;6: e24145.

20. Mooney C, Raoof R, El-Naggar H, et al. High throughput qPCR expression profiling of circulating microRNAs reveals minimal sexand sample timing-related variation in plasma of healthy volunteers. PLoS One 2015;10:e145316.

21. Tiberio $\mathrm{P}$, Callari M, Angeloni V, et al. Challenges in using circulating miRNAs as cancer biomarkers. Biomed Res Int 2015;2015:731479.

22. Khan J, Lieberman JA, Lockwood CM. Variability in, variability out: best practice recommendations to standardize pre-analytical variables in the detection of circulating and tissue microRNAs. Clin Chem Lab Med 2017;55:608-621.

23. Kim DJ, Linnstaedt S, Palma J, et al. Plasma components affect accuracy of circulating cancer-related microRNA quantitation. J Mol Diagn 2012;14:71-80

24. El-Khoury V, Pierson S, Kaoma T, et al. Assessing cellular and circulating miRNA recovery: the impact of the RNA isolation method and the quantity of input material. Sci Rep 2016;6:19529.

25. Page K, Guttery DS, Zahra N, et al. Influence of plasma processing on recovery and analysis of circulating nucleic acids. PLoS One 2013;8: e77963.

26. Farina NH, Wood ME, Perrapato SD, et al. Standardizing analysis of circulating microRNA: clinical and biological relevance. J Cell Biochem 2014;115:805-811.

27. Dellett M, Simpson DA. Considerations for optimization of microRNA PCR assays for molecular diagnosis. Expert Rev Mol Diagn 2016;16:407-414.

28. Wang Z, Gerstein M, Snyder M. RNA-Seq: a revolutionary tool for transcriptomics. Nat Rev Genet 2009;10:57-63.

29. Mills JD, Janitz M. Alternative splicing of mRNA in the molecular pathology of neurodegenerative diseases. Neurobiol Aging 2012;33:1012.e11-e24.

30. Ozsolak F, Milos PM. RNA sequencing: advances, challenges and opportunities. Nat Rev Genet 2011;12:87-98.

31. Spornraft M, Kirchner B, Haase B, et al. Optimization of extraction of circulating RNAs from plasma-enabling small RNA sequencing. PLoS One 2014:9:e107259.

32. Semenov DV, Baryakin DN, Brenner EV, et al. Unbiased approach to profile the variety of small non-coding RNA of human blood plasma with massively parallel sequencing technology. Expert Opin Biol Ther 2012;12(suppl. 1):S43-S51.

33. Huang X, Yuan T, Tschannen M, et al. Characterization of human plasma-derived exosomal RNAs by deep sequencing. BMC Genom 2013;14:319

34. Wang J, Yu JT, Tan L, et al. Genome-wide circulating microRNA expression profiling indicates biomarkers for epilepsy. Sci Rep 2015;5:9522

35. Gilad Y, Mizrahi-Man O. A reanalysis of mouse ENCODE comparative gene expression data. F1000Res 2015;4:121.

36. van Dijk EL, Jaszczyszyn Y, Thermes C. Library preparation methods for next-generation sequencing: tone down the bias. Exp Cell Res 2014:322:12-20.

37. Conesa A, Madrigal P, Tarazona S, et al. A survey of best practices for RNA-seq data analysis. Genome Biol 2016;17:13.

38. Buschmann D, Haberberger A, Kirchner B, et al. Toward reliable biomarker signatures in the age of liquid biopsies - how to standardize the small RNA-Seq workflow. Nucleic Acids Res 2016;44:5995-6018.

39. Soneson C, Delorenzi M. A comparison of methods for differential expression analysis of RNA-seq data. BMC Bioinformatics 2013;14:91.

40. Guyon I, Weston J, Barnhill S, et al. Gene selection for cancer classification using support vector machines. Mach Learn 2002;46:389-422.

41. Diaz-Uriarte R, Alvarez de Andres S. Gene selection and classification of microarray data using random forest. BMC Bioinformatics 2006;7:3.

42. Gorter JA, Iyer A, White I, et al. Hippocampal subregion-specific microRNA expression during epileptogenesis in experimental temporal lobe epilepsy. Neurobiol Dis 2014;62:508-520.

43. Roncon P, Soukupova M, Binaschi A, et al. MicroRNA profiles in hippocampal granule cells and plasma of rats with pilocarpine-induced epilepsy-comparison with human epileptic samples. Sci Rep 2015;5:14143.

44. Hu K, Zhang C, Long L, et al. Expression profile of microRNAs in rat hippocampus following lithium-pilocarpine-induced status epilepticus. Neurosci Lett 2011;488:252-257.

45. Liu DZ, Tian Y, Ander BP, et al. Brain and blood microRNA expression profiling of ischemic stroke, intracerebral hemorrhage, and kainate seizures. J Cereb Blood Flow Metab 2010;30:92-101.

46. Schroeder A, Mueller O, Stocker S, et al. The RIN: an RNA integrity number for assigning integrity values to RNA measurements. $B M C$ Mol Biol 2006;7:3.

47. Fleige S, Pfaffl MW. RNA integrity and the effect on the real-time qRT-PCR performance. Mol Aspects Med 2006;27:126-139. 
48. Gallego Romero I, Pai AA, Tung J, et al. RNA-seq: impact of RNA degradation on transcript quantification. BMC Biol 2014;12:42.

49. Becker C, Hammerle-Fickinger A, Riedmaier I, et al. mRNA and microRNA quality control for RT-qPCR analysis. Methods 2010;50:237-243.

\section{Appendix \\ Procedure for Circulating Biomarkers From RAt Plasma}

\section{Tail vein withdrawal of blood}

1 Anesthetize rat with isoflurane (5\% induction, $1-2 \%$ maintenance).

2 Warm up the tail with a heat lamp or in warm water to dilate the blood vessels. Sampling site is the lateral tail vein in the distal one-third of the tail. While extending the tail, a 23-G butterfly needle (\#367284, BD Vacutainer) is inserted into the vein and blood is collected into a $0.5-\mathrm{mL}$ $\mathrm{K}_{2}$-EDTA tube (\#365975, BD Microtainer, BD Biosciences, Franklin Lakes, NJ).

3 Blood and EDTA are mixed by inverting the tube $10 \times 180^{\circ}$ and back.

4 The EDTA tube is placed on ice after mixing and kept for a maximum of $1 \mathrm{~h}$ prior to centrifugation.

5 To separate plasma, centrifuge at $1,300 \times \boldsymbol{g}, 10 \mathrm{~min}$, $4^{\circ} \mathrm{C}$.

6 Centrifuge at $3,000 \times \mathbf{g}, 10 \mathrm{~min}, 4^{\circ} \mathrm{C}$ to remove residual platelets, preferably before freezing.

7 The sample can be kept for a maximum of $1 \mathrm{~h}$ at $4^{\circ} \mathrm{C}$. Supernatant (plasma) is pipetted into Protein LoBind tubes (\#022431064, Eppendorf) and immediately frozen on dry ice.
8 Samples are stored at $-70^{\circ} \mathrm{C}$ to $-80^{\circ} \mathrm{C}$ until processing.

\section{Plasma quality control}

1 Measure hemoglobin absorbance using a NanoDrop spectrophotometer (Thermo Scientific) at $414 \mathrm{~nm}$ using $1 \mu \mathrm{L}$ of plasma. The upper limit for absorbance is 0.25 .

\section{RNA extraction and quality control}

1 Use 50-200 $\mu \mathrm{L}$ of plasma and extract small RNAs using the miRNeasy kit (Qiagen) according to the manufacturer's instructions. For real-time quantitative polymerase chain reaction (RT-qPCR) normalization, add a (set of) spike-in template(s), e.g., Cel-miR-39.

2 Determine miRNA quality using a Fragment Analyzer (Advanced Analytical Technologies), or alternatively using a Bioanalyzer (Agilent).

3 Use a fixed volume of input material, rather than an equal quantity of RNA for RT-qPCR.

\section{Normalization RT-qPCR}

1 Use a (set of) spike-in template(s) during RNA isolation combined with a carefully selected set of assays targeting endogenous miRNAs.

\section{SUPPORTING INFORMATION}

Additional Supporting Information may be found in the online version of this article:

Table S1. Summary of blood sampling protocols in studies using rat plasma for miRNA detection. 